\title{
Do Green Building Standards Meet the Biophilic Design Strategies?
}

\author{
Beyza Şat Güngör \\ Özyeğin University, Faculty of Architecture and Design, Istanbul, Turkey
}

\author{
Received: April 28th 2020, Revised: May 19th 2020, Accepted: May 31th 2020 \\ Refer: Sat, Gungor, B., (2020), Do Green Building Standards Meet the Biophilic Design Strategies?, Journal of Design Studio, V.2, N.1, pp \\ $5-23$, \\ ORCID: 0000-0003-2579-8349 \\ DOI: $10.46474 /$ jds. 739849 . https://doi.org/10.46474/jds. 739849
}

\begin{abstract}
Energy efficiency in buildings, comprises many things as mitigation effect of global warming and climate change, decreasing heat island effect in the built environment and also conservation of natural resources. Besides as a new phenomenon we should add biophilic design criteria to the green building tools to increase human productivity by considering human wellbeing. Biophilic design, which inspired by nature, is a new juvenile design concept that gains importance day by day because of its positive effects on human wellbeing mood and relatedly human productivity. Here some conflicts can be occurred between energy saving and human wellbeing; as natural ventilation and energy saving.
\end{abstract}

Biophilic design comprises inherent human inclination to affiliate with nature. All sensations which help to be in contact with natural components as daylight, plants or some animal species like birds; plants occupy an important place in its definition. Biophilic design seeks to create good habitat for people as a biological organism in the modern built environment that promotes people's health, fitness and wellbeing. Scientific studies reveal that contact with nature has significant effects on people's physical and mental health, performance and wellbeing. This phenomenon has, an increasing importance more than ever before, especially in daily life. The need for beneficial contact with nature continues in today's built environment. This paper aims to determine whether the biophilic design strategies are match with green building tools that mostly targeting energy saving in built environment. Also paper handling whether the 14 biophilic design patterns are match with 3 main mind-body systems that are; stress reduction, cognitive performance and emotion mood preference.

Keywords: Biophilic design, natural systems, energy saving, green building tools, mind-body systems

The Term biophilia and biophilic design?

The term 'biophilia' was first used by social psychologist Eric Fromm (1964) at "The Heart of Man" and later popularized by biologist Edward Wilson (Fromm, 1964; Browing et al., 2014). The American biologist and entomologist, Edward O. Wilson, was the first to clarify the importance of contact with nature for the psychological development of people. Edward O. Wilson introduced and popularized the hypothesis in his book, "Biophilia". He defines biophilia as "the urge to affiliate with other forms of life". The term biophilia means interact with other living systems and he describes the connections that human beings subconsciously seek with the other forms of life (International Well Building Institude, 2019); for millions of years our species was related to its wild environment, created a kind of dependency, an overwhelming emotional need to be in contact with other living beings. This inherent need was called Biophilia (Wilson 1984, 1993; Sanchez et. Al. 2018). The scientists Roger S. Ulrich and Stephen Kellert gave the final approach to the term Biophilia by defining it as "the innate human affinity for nature" (Sanchez, 2018).

Although the term biophilia, named by Fromm, has been proposed and defined over many times (Browing et al. 2014), it is also defined by 
Kellert (2008) as an inherent human inclination to natural systems and processes and it urges us to affiliate with other forms of life (Kellert, 2008; Xue et al., 2019a). The concept of biophilia is the idea of human contact with nature. This connection has been thought beneficial for human physical and mental wellbeing. A study reveals the benefits of nature as healing effect (Reeve et al., 2017). As pointed out by Beatley (2016), nature is not optional, but an absolutely essential quality of modern urban life. The essential benefits of biophilia urges us not only to conserve and restore the natural elements that already exist but, insert new forms of nature for the twenty first century (Xue et al. 2019a; Beatley, 2016).

Biophilic design came in sight after the term biophilia. Alexander (2002) defines biophilic design as "integration" or sometimes "manipulation" of natural elements or systems to create sense of "life" in the built environment (Alexander, 2002). Biophilic design, defined as a response to the inherent need of human beings to be in contact with nature, improves productivity in the workplaces (Sanchez, 2018). Recently, the concept of biophilic design has received attention among practitioners and environmental psychologists (Lee, 2019). Kellert et al. (2011) explained that biophilic design inspires firms to use natural systems and processes in the design of the built environment. Biophilic design fulfills the human need for exposure to nature and several studies have shown benefits of natural features and systems into the built environment on people. Studies revealed that adding natural light and windows significantly improve participants' mood
(Kellert et al., 2011; Zadeh et al., 2014). This effect of biophilic design considered since it is related with human productivity. People's concentration increases after they spend time in nature or even view scenes of nature; reduces stress, improves cognitive functions and creativity (Lee, 2019). As the world population continues to urbanize these qualities will be ever more important (Browing et al., 2014). The United Nations predicts that by $2030,60 \%$ of the world's population will live in urban environments (UN-HABITAT, 2019).

When the biophilic design considered to increase human productivity, the studies' direction changes to follow those related subjects; Sutermeister (1976) defines performance as a quality considered output per employee hour. Accuracy and speed are the two distinct aspects to measure of human performance. Accuracy is defined as a measure of the quality of behaviour (Sanchez et al., 2018). Biophilic design modifies and apply the natural systems in the built environment for human wellbeing in many aspects and indirectly effects human performance. Biophilic spaces have been defined as spaces that strengthen life and support the sociological and psychological components (International Well Building Institute, 2019).

\section{Key strategies of biophilic design}

According to the biophilic design concept, 25 biophilic design strategies are identified by considering the related references. The key strategies of the Biophilic Design have been analysed and summarized as below table (Xue et al., 2019a).

Table 1. Key strategies of Biophilic Design (Xue et al., 2019a).

\begin{tabular}{|l|l|l|l|}
\hline $\begin{array}{l}\text { Biophilic } \\
\text { Design Aspects }\end{array}$ & Indicators & Strategies & References \\
\hline \multirow{5}{*}{$\begin{array}{l}\text { Biophilic } \\
\text { Infrastructure }\end{array}$} & Biophilia ratio & Increase green space coverage ratio; & Barton and Pretty (2010) \\
\cline { 3 - 4 } & $\begin{array}{l}\text { Promotes plant canopy configuration for } \\
\text { shading and sheltering; }\end{array}$ & Xue et al. (2017b) \\
\cline { 3 - 4 } & Enhance native species ratio; & Oldfield et al. (2015) \\
\cline { 3 - 4 } & Enhance biodiversity level; & Maes et al. (2016) \\
\hline
\end{tabular}

\footnotetext{
Journal of Design Studio, v:2 n:1
}

Sat Gungor, B., (2020), Do Green Building Standards Meet the Biophilic Design Strategies ? 


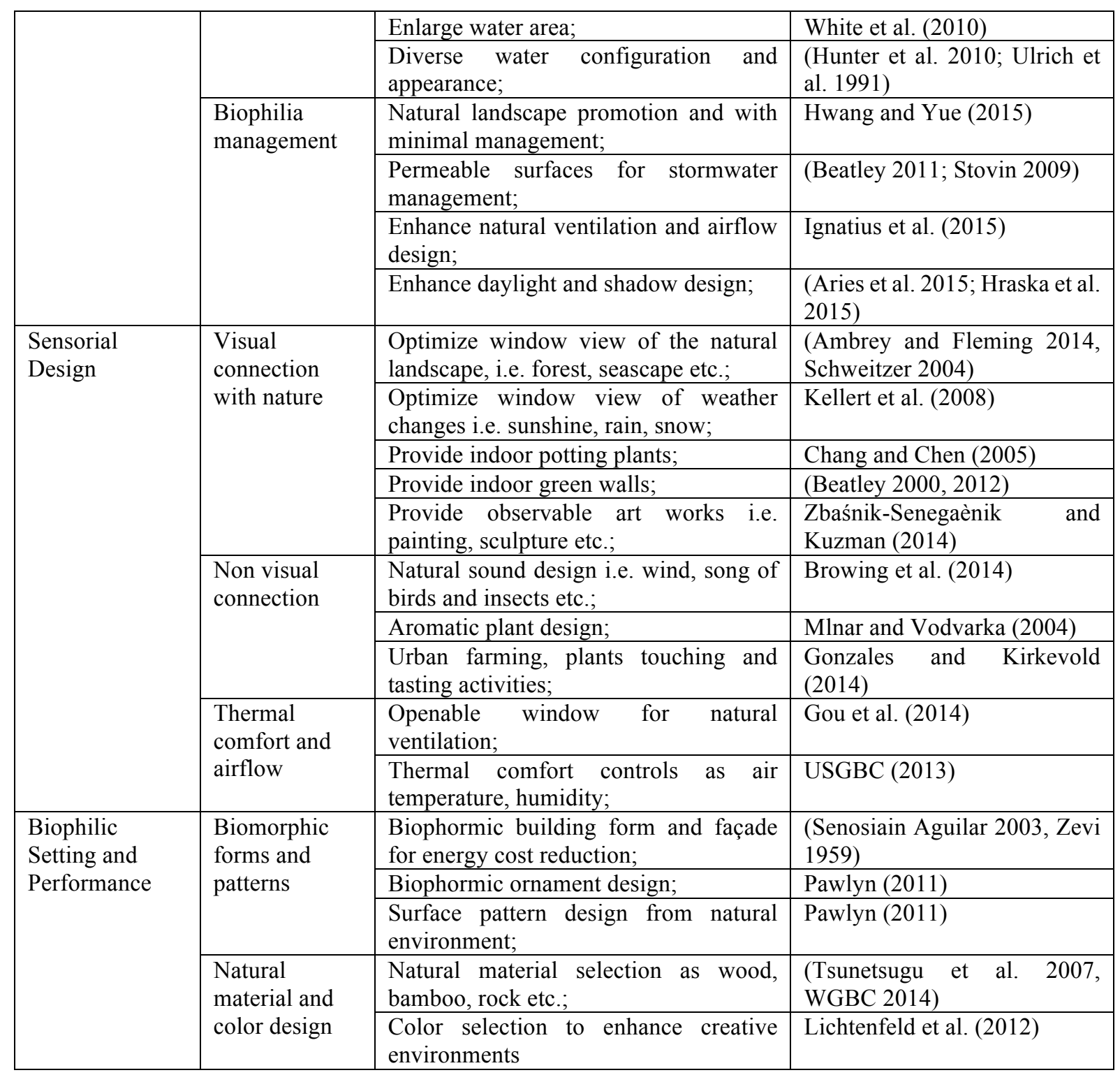

Briefly the included biophilic design strategies according to the biophilic design aspects are explained below;

Biophilic infrastructure aspect includes biophilia ratio and biophilia management; biophilia ratio is the percentage or quantity of natural elements available to users, green area cover ratio, plants canopy configuration, native species ratio, biodiversity level, and water area and appearance. Biophilia management measures the quality of the elements such as how natural landscapes require minimal management: the availability of permeable surfaces for stormwater management, the use of natural ventilation and airflow as well as the presence of daylight and shadow design.
Biophilic infrastructure is more related on quality than quantity.

Sensorial design aspect, includes visual connection, non-visual connection, thermal comfort and airflow. Visual connections with nature are the most obvious methods of biophilic design and can be achieved through having window views of natural landscapes. Non-visual connections with nature engage the other senses such as through natural sound design, aromatic plant design natural elements related art works. Natural ventilation is another factor allows people to feel in-touch with nature. 
Biophilic setting and performance aspect includes biomorphic forms and patterns and natural materials and colors. This creates a visual connection with nature with other benefits such as enhancing creativity (Xue et al., 2019a).

The visual connection with nature is an important strategy in the field of art, since implementation is relatively easier to create. Especially art works inspired by natural systems are in demand recently. This form of art combines natural daylight with natural inspired artwork. Stained glass designed by inspiration of natural cells structure, is used as the contemporary artwork in a public space, as shown in the example provided in Figure 1 and visual connection with water element that reflects light and weather conditions from above and invites by-passers to touch it, as in the example of the Luxembourg Gardens in Paris in Figure 2. Children or adults spend time by floating their wooden model sailboats and enjoy the pool's climatic effect.

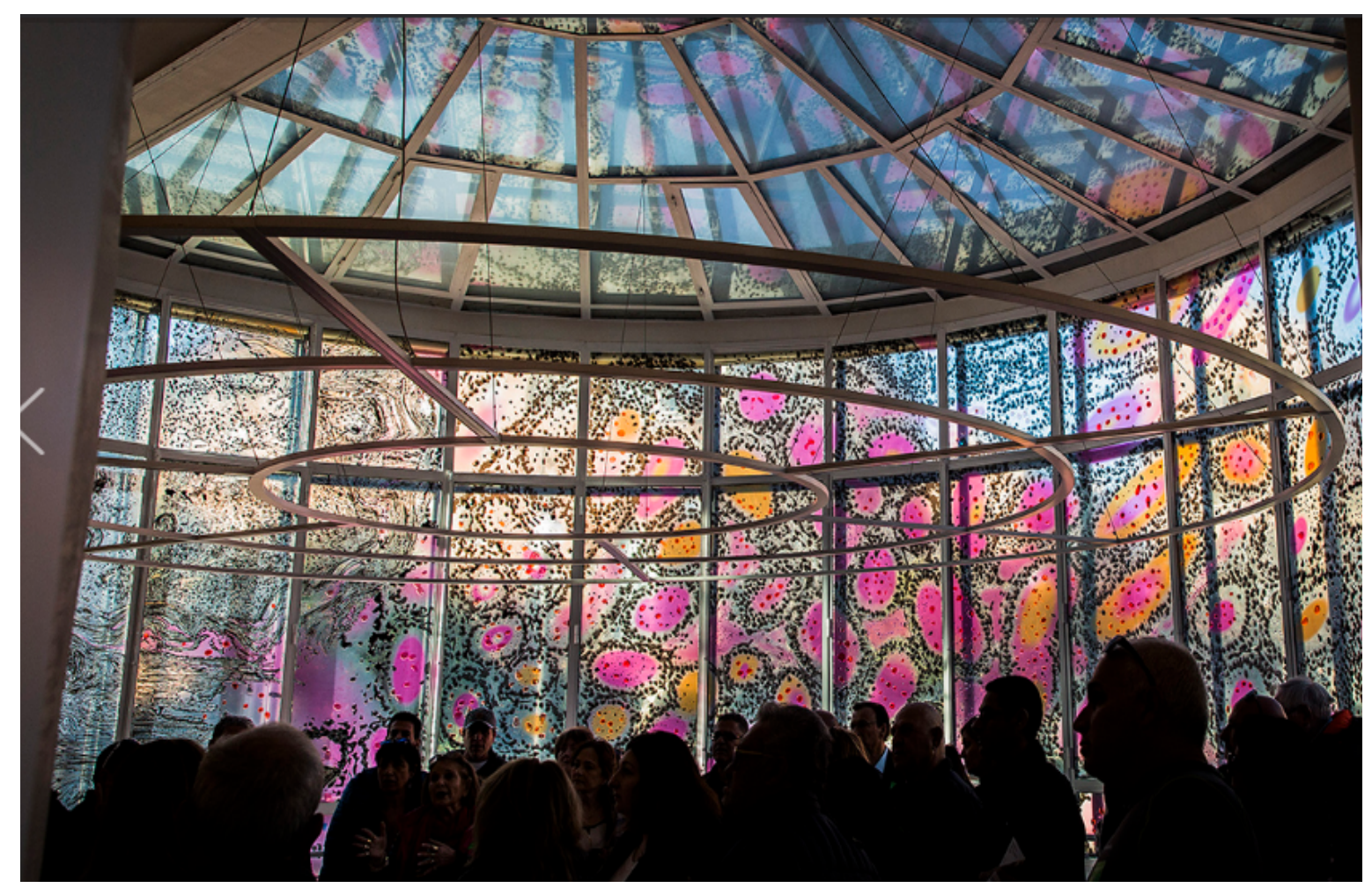

Figure 1: contemporary stained glass art work inspired from cells by Ayşe G. Süter. Photo By: Pere Virgill (Süter, 2015). 


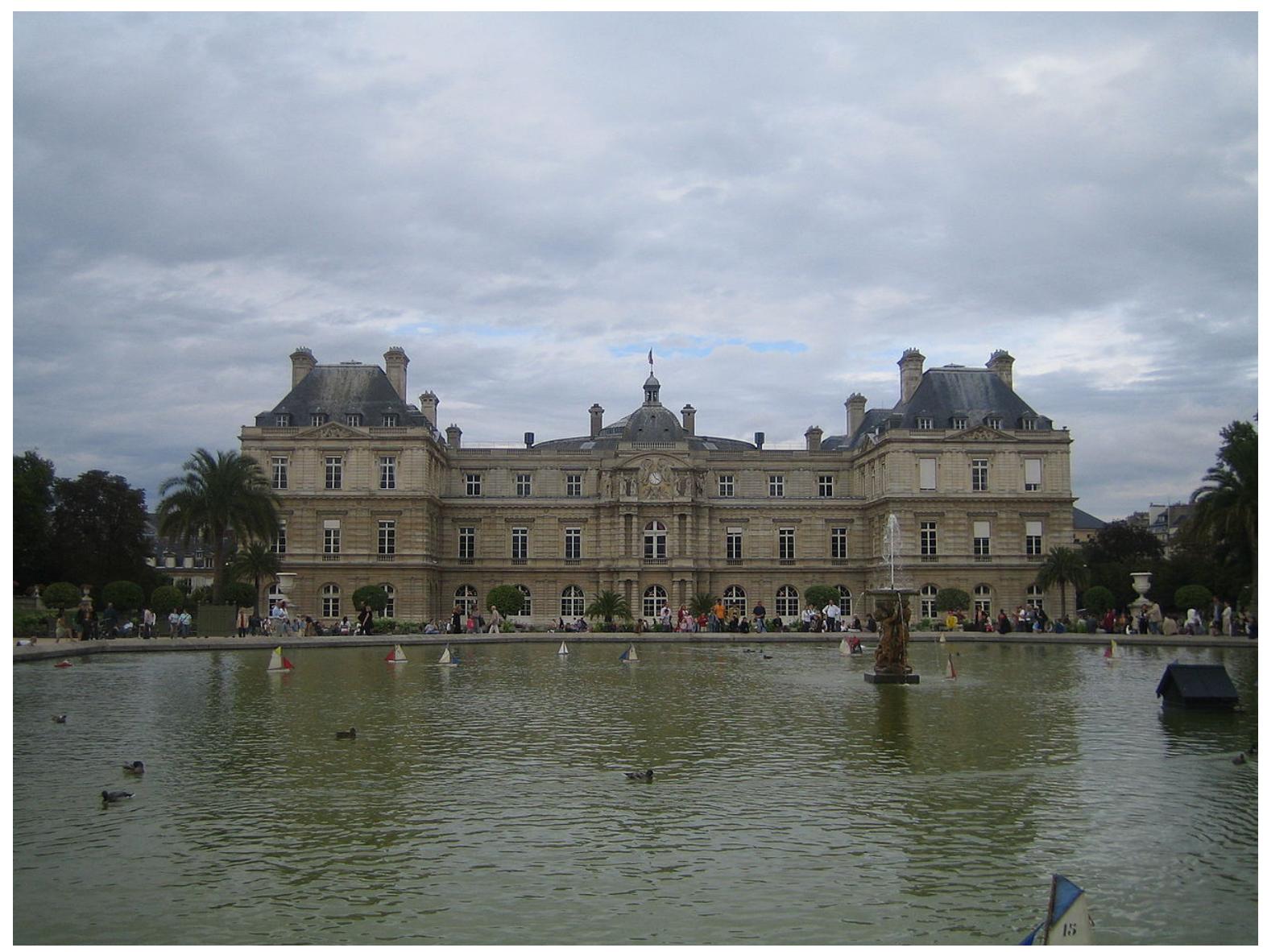

Figure 2: Water use in Luxembourg garden in Paris Photo Jiel Beaumadier CC BY-SA (Beaumadier, 2006).

\section{Biophilic design strategies and Green Building Ratio Tools (GBRTs)}

Green Building Ratios are an indicator to measure green architecture. They are more focused on energy efficiency. How can we measure the biophilic designed space meets human wellbeing and improve productivity? This question's answer is a challenge. Actually there are many indoor stressors effective on human wellbeing and productivity, such as excessive thermal factors, lighting aspects, moisture, noise and vibration, radiation, chemical compounds, and particle fluctuations. In a place, a whole range of effects has been associated with these stressors that known as Sick Building Syndrome (SBS) (Sanchez et al., 2018). A research implemented in Keio University - which focused on the element that is most valued at the work environment - shows that in top five natural light was the most valued element, followed by indoor plants and vivid colours. Use of daylight is most effective on reducing the fatigue feeling.
Biophilic design strategies are added to the principles of Green architecture. In fact, biophilic design includes some of the green building standards but it targets not only energy saving but also human wellbeing, good mood and productivity. Biophilic strategies and their sub-relevant categories are matched with updated Green Building Rating Tools (GBRTs) as LEED (Leadership in Energy and Environmental Design), BREEAM (Building Research Establishment Environmental Assessment) GM NRB (Green Mark NonResidential Building), GBL (Green Building Label), WBS (Well Building Standard) and LBC (Living Building Challenge). The framework is used in a recent study to emphasize the shifting of GBRTs from the energy-oriented approach to a human-oriented approach through biophilic strategies (Xue et al., 2019b). 
Table 2: Selected GBRTs

\begin{tabular}{|l|l|}
\hline Selected GBRTs & Classification \\
\hline LEED & $\begin{array}{l}\text { Internationally most widespread, industry standard for sustainability (Shan and Hwang, } \\
\text { 2018; Xie and Gou, 2017). }\end{array}$ \\
\cline { 1 - 2 } BREEAM & $\begin{array}{l}\text { Developed and employed in the local situations and are tailored to native climates and } \\
\text { contexts: respectively China and Singapore (Hwang et al., 2016; Shan and Hwang, } \\
\text { 2018; Ye et al., 2015; Zou et al., 2017). }\end{array}$ \\
\cline { 1 - 2 } GM & $\begin{array}{l}\text { Innovative green building regulations which focus on human health and wellbeing. First } \\
\text { protocol to address human wellness into the built environment (Morton, 2015). }\end{array}$ \\
\hline WBS &
\end{tabular}

Each GBRT credit has been assessed in terms of its intent to improve human health and wellbeing. Some credits such as thermal comfort and air quality are dedicated to improving human health; these credits are easily identified as human-oriented credits. Some credits such as public transport and cycling have multi-purposes including reducing greenhouse gas emissions while improving health and wellbeing. These credits are also human-oriented credits. Other credits that are dedicated to energy efficiency and greenhouse gas emission reduction are excluded. Prominently, WBS and LBC have more credits on human health and wellbeing. The GBRT credits are generally grouped in 8 categories: Place, Transport, Energy, IEQ (Indoor Environmental Quality), Water, Material, Health and Management. The relationship between the 8 categories of GBRT credits and the 6 different GBRT's are analysed in a crosstable by considering biophilic design strategies (Xue et al., 2019b).

As seen in Table 3.1 the biophilic infrastructure deals with essential comfortable conditions for human wellbeing as natural ventilation and day light.

Table 3.1: GBRTs matching with "biophilic infrastructure" design category and strategies (Xue et al., 2019b).

\begin{tabular}{|l|l|l|l|l|l|l|}
\hline Strategies & LEED & BREEAM & GM & GBL & WBS & LBC \\
\hline $\begin{array}{l}\text { Increase } \\
\text { green space } \\
\text { coverage } \\
\text { ratio; }\end{array}$ & Place & & & & & \\
\hline $\begin{array}{l}\text { Promote } \\
\text { plants canopy } \\
\text { configuration } \\
\text { for shading } \\
\text { and } \\
\text { sheltering; }\end{array}$ & Place & Place & Place & Place & Place & \\
\hline $\begin{array}{l}\text { Enhance } \\
\text { native } \\
\text { species ratio; }\end{array}$ & Place & Water & Place & Place & Place & \\
\hline $\begin{array}{l}\text { Enhance } \\
\text { biodiversity } \\
\text { level; }\end{array}$ & & & & & & \\
\hline $\begin{array}{l}\text { Enlarge } \\
\text { water area; }\end{array}$ & & Place & Place & Place & & \\
\hline $\begin{array}{l}\text { Diverse } \\
\text { water } \\
\text { configuration } \\
\text { and } \\
\text { appearance; }\end{array}$ & & & & & Place \\
\hline
\end{tabular}




\begin{tabular}{|c|c|c|c|c|c|c|}
\hline $\begin{array}{l}\text { Natural } \\
\text { landscape } \\
\text { promotion } \\
\text { with minimal } \\
\text { management; }\end{array}$ & Water & Place & Place & Place & & Place \\
\hline $\begin{array}{l}\text { Permeable } \\
\text { surfaces for } \\
\text { storm water } \\
\text { management; }\end{array}$ & Place & Water & Place & Place & & Water \\
\hline $\begin{array}{l}\text { Enhance } \\
\text { natural } \\
\text { ventilation } \\
\text { and airflow } \\
\text { design; }\end{array}$ & IEQ & IEQ & IEQ & IEQ & IEQ & IEQ \\
\hline $\begin{array}{l}\text { Enhance } \\
\text { daylight and } \\
\text { shadow } \\
\text { design; }\end{array}$ & IEQ & IEQ & IEQ & IEQ & IEQ & IEQ \\
\hline
\end{tabular}

"Enlarge water area" biophlic infrastructure design category does not match any of the GBRTs and "Diverse water configuration and appearance" category just match with two of the GBRTs. In this table we can summarize just three of the credits as water, place and IEQ related with biophilic infrastructure design category.

Table 3.2 presents the sensorial design category of biophilic design strategies that match with GBRT credits like Place, IEQ (Indoor Environmental Quality) and Health.

Table 3.2: GBRTs matching with "sensorial design" design category and strategies (Xue et al., 2019b).

\begin{tabular}{|c|c|c|c|c|c|c|}
\hline Strategies & LEED & BREEAM & GM & GBL & WBS & LBC \\
\hline $\begin{array}{l}\text { Optimize } \\
\text { window view of } \\
\text { the natural } \\
\text { landscape. i.e. } \\
\text { forest, seascape, } \\
\text { water motif, } \\
\text { etc.; }\end{array}$ & IEQ & IEQ & & IEQ & IEQ & IEQ \\
\hline $\begin{array}{l}\text { Optimize } \\
\text { window view of } \\
\text { weather changes } \\
\text { (i.e. the } \\
\text { sunshine, rain, } \\
\text { snow); }\end{array}$ & IEQ & IEQ & & & IEQ & \\
\hline $\begin{array}{l}\text { Provide indoor } \\
\text { potting plants; }\end{array}$ & & & Health & & Health & \\
\hline $\begin{array}{l}\text { Provide indoor } \\
\text { green walls; }\end{array}$ & & & Health & IEQ & Health & \\
\hline $\begin{array}{l}\text { Provide } \\
\text { observable } \\
\text { artworks (i.e. } \\
\text { painting, } \\
\text { sculpture); }\end{array}$ & & & Health & & Health & Health \\
\hline
\end{tabular}




\begin{tabular}{|l|l|l|l|l|l|l|}
\hline $\begin{array}{l}\text { Natural sound } \\
\text { design (i.e. the } \\
\text { wind, song of } \\
\text { birds and } \\
\text { insects); }\end{array}$ & & & & & & \\
\hline $\begin{array}{l}\text { Aromatic plant } \\
\text { design (i.e. } \\
\text { certain trees and } \\
\text { flowers); }\end{array}$ & & & & & Health & \\
\hline $\begin{array}{l}\text { Urban farming } \\
\text { (i.e. plants- } \\
\text { touching and } \\
\text { tasting } \\
\text { activities); }\end{array}$ & & & & & & \\
\hline $\begin{array}{l}\text { Openable } \\
\text { window for } \\
\text { natural } \\
\text { ventilation; }\end{array}$ & Place & & & & & \\
\hline $\begin{array}{l}\text { Individual/group } \\
\text { thermal comfort } \\
\text { controls (i.e. air } \\
\text { temperature, air } \\
\text { speed, and }\end{array}$ & & & & & & \\
\hline
\end{tabular}

"Sensorial design" category includes only three credits as IEQ, health and place. Place has seen just at LBC tool. Health and IEQ seen as more related with sensorial design category in biophilic design strategies.
Table 3.3. presents the relation to the built environment, through materials, textures, forms and structures. It is at this level where energy efficiency provided by the thermal insulation and ventilation is tackled.

Table 3.3: GBRTs matching with "Biophilic setting and performance" design category and strategies (Xue et al., 2019b).

\begin{tabular}{|l|l|l|l|l|l|l|}
\hline Strategies & LEED & BREEAM & GM & GBL & WBS & LBC \\
\hline $\begin{array}{l}\text { Biomorphic } \\
\text { building form and } \\
\text { façade for energy } \\
\text { cost reduction; }\end{array}$ & & & & & & \\
\hline $\begin{array}{l}\text { Biomorphic } \\
\text { ornament design } \\
\text { (i.e. Golden mean } \\
\text { and Fibonacci } \\
\text { series); }\end{array}$ & & & Health & & & \\
\hline $\begin{array}{l}\text { Surface pattern } \\
\text { design from } \\
\text { natural } \\
\text { environment (i.e. } \\
\text { pattern of animal } \\
\text { skin); }\end{array}$ & & & Health & & & Health \\
\hline $\begin{array}{l}\text { Natural material } \\
\text { selection, i.e. } \\
\text { wood, bamboo, } \\
\text { rock, etc.; }\end{array}$ & Material & Material & Material & Material & Material & Material \\
\hline
\end{tabular}




\begin{tabular}{|l|l|l|l|l|l|l|}
\hline $\begin{array}{l}\text { Color selection to } \\
\text { enhance creative } \\
\text { environments. }\end{array}$ & Health & & & & & \\
\hline
\end{tabular}

"Biophilic setting and performance" category includes mainly health and material credits in biophilic design strategies. Mainly GM and LBC matches.
Table 3.4 emphasized the fact that Place, Health and IEQ (Indoor Environmental Quality) credits are dominant in the transportation connectivity category of biophilic design strategies.

Table 3.4: GBRTs matching with "transportation connectivity" design category and strategies (Xue et al., 2019b).

\begin{tabular}{|c|c|c|c|c|c|c|}
\hline Strategies & LEED & BREEAM & GM & GBL & WBS & LBC \\
\hline $\begin{array}{lr}\text { Locate } & \text { public } \\
\text { bus/MRT } & \text { station } \\
\text { within } & 300 \mathrm{~m} \\
\text { walking } & \\
\text { distance; } & \\
\end{array}$ & Transport & Transport & & Transport & Transport & Transport \\
\hline $\begin{array}{ll}\text { Provide a fully } \\
\text { connected } \\
\text { pedestrian } \\
\text { network; }\end{array}$ & Transport & Transport & & & Transport & \\
\hline $\begin{array}{l}\text { Provide shaded } \\
\text { corridors/bridges } \\
\text { between } \\
\text { buildings and } \\
\text { districts; }\end{array}$ & & & & & & \\
\hline $\begin{array}{lr}\text { Provide fully } \\
\text { accessible and } \\
\text { dedicated } \\
\text { cycling lanes; }\end{array}$ & Transport & Transport & Transport & Transport & Place & \\
\hline $\begin{array}{l}\text { Configure with } \\
\text { bike parking } \\
\text { facilities; }\end{array}$ & Transport & Transport & Transport & Transport & Place & Place \\
\hline $\begin{array}{l}\text { Configure with } \\
\text { change room and } \\
\text { shower facilities; }\end{array}$ & Transport & Transport & Transport & & Place & Place \\
\hline $\begin{array}{lr}\text { Provide } & \\
\text { landmark } & \text { in } \\
\text { public } & \text { open } \\
\text { space } & \text { for } \\
\text { attraction } & \text { and } \\
\text { gathering; } & \\
\end{array}$ & & & & & & \\
\hline $\begin{array}{l}\text { Effective visual } \\
\text { information } \\
\text { system design } \\
\text { for wayfinding } \\
\text { and } \\
\text { collaboration; }\end{array}$ & Place & Transport & & & Place & \\
\hline $\begin{array}{lr}\text { Locate } & \text { public } \\
\text { bus/MRT } & \text { station } \\
\text { within } & 300 \mathrm{~m} \\
\text { walking } & \\
\text { distance; } & \\
\end{array}$ & Transport & Transport & & Transport & Transport & Transport \\
\hline
\end{tabular}


"Transportation connectivity" category includes just transport and place credits in biophilic design strategies match. GM and LBC seen weaker in this category match.
Table 3.5 indicates "place" and "management" credits that meet with the work-live-play integration category of the biophilic design strategy.

Table 3.5: GBRTs matching with "work-live-play integration" design category and strategies (Xue et al., 2019b).

\begin{tabular}{|l|l|l|l|l|l|l|}
\hline Strategies & LEED & BREEAM & GM & GBL & WBS & LBC \\
\hline $\begin{array}{l}\text { Share public } \\
\text { green spaces, } \\
\text { open plaza } \\
\text { and } \\
\text { community } \\
\text { spaces; }\end{array}$ & & & & & & \\
\hline $\begin{array}{l}\text { Share food \& } \\
\text { beverage, } \\
\text { food court } \\
\text { facilities; }\end{array}$ & Place & Management & Management & Place & Management & Place \\
\hline $\begin{array}{l}\text { Share } \\
\text { learning and } \\
\text { collaboration } \\
\text { facilities; }\end{array}$ & Place & Management & & & & \\
\hline $\begin{array}{l}\text { Security } \\
\text { management } \\
\text { (i.e. patrol, } \\
\text { CCTV); }\end{array}$ & & & & & & \\
\hline $\begin{array}{l}\text { Enhance } \\
\text { facility and } \\
\text { site } \\
\text { maintenance; }\end{array}$ & Management & Management & Management & Management & Management & Management \\
\hline
\end{tabular}

"Work-live-play" integration category includes just management and place credits in biophilic design strategies match again GM and LBC seen weak in this category match. "Green space place making" category mostly matches with WBS and includes just place and management credits.
Most of the biophilic strategies $(\% 85)$ are matching with GBRTs. 5 biophilic strategies that are presented below does not match any of the GBRTs;

- enlarge water area,

- aromatic plant design,

- shaded corridors/bridges between buildings and districts,

- landmark in public open space for attraction and gathering

- shaded outdoor seats for café and restaurants. 
Table 3.6: GBRTs matching with "green space place making" design category and strategies (Xue et al., 2019b).

\begin{tabular}{|l|l|l|l|l|l|l|}
\hline Strategies & $\begin{array}{l}\text { LEE } \\
\text { D }\end{array}$ & BREEAM & GM & GBL & WBS & LBC \\
\hline $\begin{array}{l}\text { Provide } \\
\text { outdoor seats for café } \\
\text { and restaurants; }\end{array}$ & & & & & & \\
\hline $\begin{array}{l}\text { Volunteer-engagement } \\
\text { for urban farming; }\end{array}$ & & & & & Place & Place \\
\hline $\begin{array}{l}\text { Provide Friday/holiday } \\
\text { market (vegetable, } \\
\text { food, and other } \\
\text { commercial sales); }\end{array}$ & Place & & & & & \\
\hline $\begin{array}{l}\text { Provide outdoor } \\
\text { performance/exhibitio } \\
\text { n of art events during } \\
\text { lunch breaks (i.e. live }\end{array}$ & & $\begin{array}{l}\text { Managemen } \\
\mathrm{t}\end{array}$ & $\begin{array}{l}\text { Managemen } \\
\mathrm{t}\end{array}$ & $\begin{array}{l}\text { Managemen } \\
\mathrm{t}\end{array}$ & $\begin{array}{l}\text { Managemen } \\
\mathrm{t}\end{array}$ & $\begin{array}{l}\text { Managemen } \\
\mathrm{t}\end{array}$ \\
\hline
\end{tabular}

\section{Biophilic patterns and mind-body systems}

Mind-body systems that relate directly with people's health and well-being are impacted by the environment. Table 4 clarifies the relationships between 14 biophilic design patterns and mind-body systems with related references. Biophilic design addresses to people as a biological organism, respecting the mind-body systems as indicators of health and well-being. There are three factors identified in mind-body systems. Those considered main mind-body systems are; stress reduction, cognitive performance and emotion mood preference.

Table 4: 14 Biophilic Design Patterns matching with Mind-Body Systems (Browing et al., 2014).

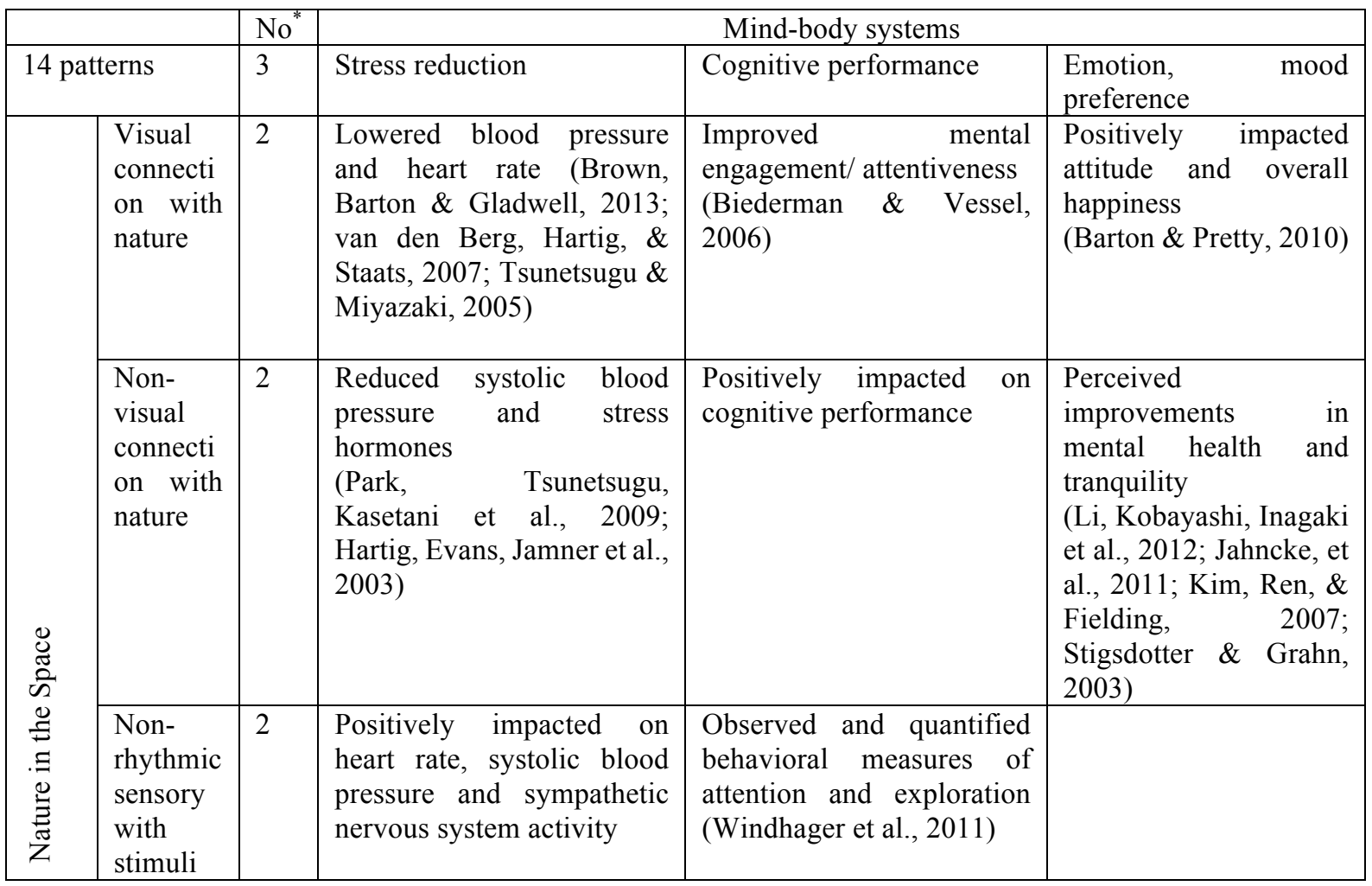




\begin{tabular}{|c|c|c|c|c|c|}
\hline & & & $\begin{array}{l}\text { (Kahn et al., 2008; } \\
\text { Beauchamp, et al., 2003) }\end{array}$ & & \\
\hline & $\begin{array}{l}\text { Thermal } \\
\& \\
\text { Airflow } \\
\text { with } \\
\text { variabilit } \\
\text { y }\end{array}$ & 2 & $\begin{array}{l}\text { Positively impacted } \\
\text { comfort, well-being and } \\
\text { productivity } \\
\text { (Heerwagen, 2006; Tham \& } \\
\text { Willem, 2005; Wigö, 2005) }\end{array}$ & $\begin{array}{l}\text { Positively impacted } \\
\text { concentration } \\
\text { (Hartig et al., 2003; Hartig } \\
\text { et al., 1991; R. Kaplan \& } \\
\text { Kaplan, 1989) }\end{array}$ & $\begin{array}{l}\text { Improved perception of } \\
\text { temporal and spatial } \\
\text { pleasure (alliesthesia) } \\
\text { (Parkinson, de Dear \& } \\
\text { Candido, 2012; Zhang, } \\
\text { Arens, Huizenga \& } \\
\text { Han, 2010; Arens, } \\
\text { Zhang \& Huizenga, } \\
\text { 2006; Zhang, 2003; de } \\
\text { Dear \& Brager, 2002; } \\
\text { Heschong, 1979) }\end{array}$ \\
\hline & $\begin{array}{l}\text { Presence } \\
\text { of Water }\end{array}$ & 2 & $\begin{array}{l}\text { Reduced stress, increased } \\
\text { feelings of tranquility, } \\
\text { lower heart rate and blood } \\
\text { pressure } \\
\text { (Alvarsson, Wiens, \& } \\
\text { Nilsson, 2010; Biederman } \\
\text { \& Vessel, 2006) }\end{array}$ & $\begin{array}{l}\text { Improved concentration } \\
\text { and memory restoration } \\
\text { (Alvarsson et al., 2010; } \\
\text { Biederman \& Vessel, } \\
\text { 2006) } \\
\text { Enhanced perception and } \\
\text { psychological } \\
\text { responsiveness } \\
\text { (Alvarsson et al., 2010; } \\
\text { Hunter et al., 2010) }\end{array}$ & $\begin{array}{l}\text { Observed preferences } \\
\text { and positive emotional } \\
\text { responses } \\
\text { (Windhager, } \\
\text { Barton \& Pretty, 2011; } \\
\text { White, Smith, } \\
\text { Humphryes et al., 2010; } \\
\text { Karmanov \& Hamel, } \\
\text { 2008; Biederman \& } \\
\text { Vessel, } \\
\text { Heerwagen \& Orians, } \\
\text { 1993; Ruso } \\
\text { Atzwanger, } \\
\text { Ulrich, 1983) }\end{array}$ \\
\hline & $\begin{array}{l}\text { Dynami } \\
\mathrm{c} \quad \& \\
\text { Diffuse } \\
\text { light }\end{array}$ & 2 & $\begin{array}{l}\text { Positively impacted } \\
\text { circadian } \\
\text { functioning } \\
\text { (Figueiro, Brons, Plitnick et } \\
\text { al., 2011; Beckett \& Roden, } \\
\text { 2009) } \\
\text { Increased visual comfort } \\
\text { (Elyezadi, 2012; Kim \& } \\
\text { Kim, 2007) }\end{array}$ & & \\
\hline & $\begin{array}{l}\text { Connecti } \\
\text { on with } \\
\text { natural } \\
\text { systems }\end{array}$ & & & & $\begin{array}{l}\text { Enhanced positive } \\
\text { health responses; } \\
\text { Shifted perception of } \\
\text { environment } \\
\text { (Kellert et al., } 2008 \\
\end{array}$ \\
\hline & $\begin{array}{l}\text { Biophor } \\
\text { mic } \\
\text { Forms \& } \\
\text { Patterns }\end{array}$ & 1 & & & $\begin{array}{ll}\text { Observed } & \text { view } \\
\text { preference } & \\
\text { (Vessel, 2012; Joye, } \\
\text { 2007) }\end{array}$ \\
\hline 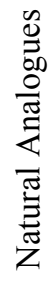 & $\begin{array}{l}\text { Material } \\
\text { Connecti } \\
\text { on with } \\
\text { Nature }\end{array}$ & & & $\begin{array}{l}\text { Decreased diastolic blood } \\
\text { pressure } \\
\text { (Tsunetsugu, Miyazaki \& } \\
\text { Sato, 2007) } \\
\text { Improved creative } \\
\text { performance } \\
\text { (Lichtenfeld et al., 2012) }\end{array}$ & $\begin{array}{l}\text { Improved comfort } \\
\text { (Tsunetsugu, Miyazaki } \\
\text { \& Sato 2007) }\end{array}$ \\
\hline & $\begin{array}{l}\text { Comple } \\
\text { xity \& } \\
\text { Order }\end{array}$ & 2 & $\begin{array}{lr}\text { Positively } & \text { impacted } \\
\text { perceptual } & \text { and } \\
\text { physiological } & \text { stress } \\
\text { responses } & \end{array}$ & & $\begin{array}{lr}\text { Observed } & \text { view } \\
\text { preference } & \\
\text { (Salingaros, } & 2012 ; \\
\text { Hägerhäll, } & \text { Laike, }\end{array}$ \\
\hline
\end{tabular}




\begin{tabular}{|c|c|c|c|c|c|}
\hline & & & $\begin{array}{l}\text { (Salingaros, 2012; Joye, } \\
\text { 2007; Taylor, 2006; S. } \\
\text { Kaplan, 1988) }\end{array}$ & & $\begin{array}{l}\text { Taylor et al., 2008; } \\
\text { Hägerhäll, Purcella, \& } \\
\text { Taylor, 2004; Taylor, } \\
\text { 2006) }\end{array}$ \\
\hline \multirow{4}{*}{ 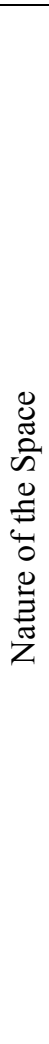 } & Prospect & 3 & $\begin{array}{l}\text { Reduced stress } \\
\text { (Grahn \& Stigsdotter, 2010) }\end{array}$ & $\begin{array}{lr}\text { Reduced } & \text { boredom, } \\
\text { irritation, } & \text { fatigue } \\
\text { (Clearwater \& } & \text { Coss, 1991) }\end{array}$ & $\begin{array}{l}\text { Improved comfort and } \\
\text { perceived } \\
\text { (Herzog \& } \text { safety } \\
\text { 2007; Wang \& Taylor, } \\
\text { 2006; Petherick, 2000) }\end{array}$ \\
\hline & Refuge & 3 & & $\begin{array}{l}\text { Improved concentration, } \\
\text { attention and perception of } \\
\text { safety } \\
\text { (Grahn \& Stigsdotter, } \\
\text { 2010; Wang \& Taylor, } \\
\text { 2006; Wang \& Taylor, } \\
\text { 2006; Petherick, 2000) }\end{array}$ & \\
\hline & Mystery & 2 & & & $\begin{array}{l}\text { Induced strong pleasure } \\
\text { response } \\
\text { (Biederman, 2011; } \\
\text { Salimpoor, Benovoy, } \\
\text { Larcher et al., 2011; } \\
\text { Ikemi, 2005; Blood \& } \\
\text { Zatorre, 2001) }\end{array}$ \\
\hline & $\begin{array}{l}\text { Risk/Per } \\
\text { il }\end{array}$ & 1 & & & $\begin{array}{l}\text { Resulted in strong } \\
\text { dopamine or pleasure } \\
\text { responses } \\
\text { (Kohno et al., 2013; } \\
\text { Wang \& Tsien, 2011; } \\
\text { Zald et al., 2008) }\end{array}$ \\
\hline
\end{tabular}

\section{Discussion and Conclusion}

When we examine the GBRT's with biophilic design strategies; it is obvious that the GBRT's are insufficient to meet the needs of biophilic design. New approaches and additions should be provided to meet those strategies in the concept of human wellbeing, good mood and productivity. In the other hand it is obvious that biophilic patterns are effective on mind-body systems positively. However, it needs more empirical data on that. Some nature interactions can even induce stress or fear responses. Fortunately, an emerging number of studies teach us which specific interactions with nature are restorative and which are stressful. Understanding how people viscerally respond to nature and how such beneficial experiences can be supported in urban settings is essential to shaping a healthy and vibrant society. Biophilic design must be implemented correctly to optimize health benefits (Ryan, 2014).
The therapeutic influence of the natural environment on human is being lost, touch of nature into our daily lives can raise this again. The scientific results that supporting biophilic design is still emerging. Deep down, we know that the connection to nature is important. While empirical evidence is accumulating, we ought to go about restoring the human-nature connection in the built environment. In coming decades, it is projected that 70 percent of the world's population will live in cities. It makes human more be in search of nature to feel fresh. This is estimated that biophilic design will get more important day by day. Biophilic Design helps shed light on the importance of the human connections with nature in our built environment and encourages people to challenge convention by bringing biophilic design patterns into a vision for healthy homes, workplaces and cities. 


\section{References}

Alexander, C. (2002). The Nature of Order. Center for Environmental Structure. Berkeley, California. Beatley, T. (2016). Handbook of Biophilic City Planning and Design. Island Press, Washington D.C.

Alvarsson, J., S. Wiens \& M. Nilsson (2010). Stress Recovery during Exposure to Nature Sound and Environmental Noise. International Journal of Environmental Research and Public Health, 7 (3), 1036-1046.

Ambrey, C., Fleming, C. (2014). Public greenspace and life satisfaction in urban Australia. Urban Stud. 51, 1290e1321.

Arens, E., H. Zhang, \& C. Huizenga (2006). Partialand Whole-body Thermal Sensation and Comfort, Part II: Non-uniform Environmental Conditions. Journal of Thermal Biology, 31, 60-66.

Aries, M.B.C., Aarts, M.P.J., van Hoof, J. (2015). Daylight and health: a review of the evidence and consequences for the built environment. Light. Res. Technol. 47, 6e27.

Barton, J., Pretty, J. (2010). What is the best dose of nature and green exercise for improving mental health? A multi-study analysis. Environ. Sci. Technol. 44, 3947e3955.

Beaumadier, J. (2006). [photograph]. Palais du Luxemburg, Paris. Retrieved fromhttps://en.wikipedia.org/wiki/Jardin_du_Luxe mbourg\#/media/File:Jielbeaumadier_palais__du_lux embourg_paris_2006.jpg

Beatley, T. (2011). Biophilic Cities: Integrating Nature into Urban Design and Planning. Island Press, Washington, DC.

Beatley, T. (2000). Green Urbanism: Learning from European Cities. Island Press, Washington DC.

Beatley, T. (2012). Singapore: how to grow a highrise city in a garden. Site Lines $8,14 \mathrm{e} 17$.

Beauchamp, M.S., K.E. Lee, J.V. Haxby,\& A. Martin (2003). FMRI Responses To Video and Point-Light Displays of Moving Humans and Manipulable Objects. Journal of Cognitive Neuroscience, 15 (7), 991-1001.

Beckett, M. \& L.C. Roden (2009). Mechanisms by which circadian rhythm disruption may lead to cancer. South African Journal of Science 105, November/December 2009.

Biederman, I. \& E. Vessel (2006). Perceptual Pleasure \& the Brain. American Scientist, 94(1), 249-255.

Biederman, I. (2011). University of Southern California, Department of Psychology. Personal communication with the authors.

Blood, A., \& R.J. Zatorre (2001). Intensely Pleasurable Responses to Music Correlate with Activity in Brain Regions. Proceedings from the National Academy of Sciences, 98 (20), 1181811823.

Brown, D.K., J.L. Barton, \& V.F. Gladwell (2013). Viewing Nature Scenes Positively Affects Recovery of Autonomic Function Following Acute-Mental Stress. Environmental Science \& Technology, 47, 5562-5569.

Browning, W.D., Ryan, C.O., Clancy, J.O. (2014). 14 Patterns of Biophilic Design. New York: Terrapin Bright Green llc.

Browning, W., Ryan, C., Clancy, J. (2014). 14 Patterns of Biophilic Design, Improving Health \& Well-being in the Built Environment. Terrapin Bright Green LLC, New York, p. 62.

Chang, C.-Y., Chen, P.-K. (2005). human response to window views and indoor plants in the workplace. In: HortScience, vol. 40, pp. 1354e1359.

Clearwater, Y.A., \& R.G. Coss (1991). Functional Esthetics to Enhance Wellbeing. In Harrison, Clearwater \& McKay (Eds.). From Antarctica to Outer Space. New York: Springer-Verlag, pp410.

de Dear, R. \& G. Brager (2002). Thermal comfort in naturally ventilated buildings. Energy and Buildings, 34, 549-561.

Elzeyadi, I.M.K. (2012). Quantifying the Impacts of Green Schools on People and Planet. Research presented at the USGBC Greenbuild Conference \& Expo, San Francisco, November 2012, 48-60.

Figueiro, M.G., J.A. Brons, B. Plitnick, B. Donlan, R.P. Leslie, \& M.S. Rea (2011). Measuring circadian light and its impact on adolescents. Light Res Technol. 43 (2): 201-215.

Fromm, E. (1964). The Heart of Man. Harper \& Row 
Gonzalez, M.T., Kirkevold, M. (2014). Benefits of sensory garden and horticultural activities in dementia care: a modified scoping review. J. Clin. Nurs. 23, 2698e 2715.

Gou, Z., Prasad, D., Lau, S.S.-Y. (2014). Impacts of green certifications, ventilation and office types on occupant satisfaction with indoor environmental quality. Architect. Sci. Rev. 57, $196 \mathrm{e} 206$.

Grahn, P. \& U.K. Stigsdotter (2010). The Relation Between Perceived Sensory Dimensions of Urban Green Space and Stress Restoration. Landscape and Urban Planning 94, 264-275.

Hartig, T., M. Mang, \& G. W. Evans (1991). Restorative Effects of Natural Environment Experience. Environment and Behavior, 23, 3-26.

Hartig, T., G.W. Evans, L.D. Jamner, D.S. Davis, \& T. Gärling (2003). Tracking Restoration in Natural and Urban Field Settings. Journal of Environmental Psychology, 23, 109-123.

Heerwagen, J.H. (2006). Investing In People: The Social Benefits of Sustainable Design. Rethinking Sustainable Construction. Sarasota, FL. September 19-22, 2006.

Heerwagen, J.H. \& G.H. Orians (1993). Humans, Habitats and Aesthetics. In: S.R. Kellert \& R.S. Wilson (Eds.). The Biophilia Hypothesis (138-172). Washington: Island Press. pp484.

Heschong, L. (1979). Thermal Delight in Architecture. Cambridge, MA: MIT Press.

Herzog, T.R. \& A.G. Bryce (2007). Mystery and Preference in Within-Forest Settings. Environment and Behavior, 39 (6), 779-796.

Hägerhäll, C.M., T. Laike, R. P. Taylor, M. Küller, R. Küller, \& T. P. Martin (2008). Investigations of Human EEG Response to Viewing Fractal Patterns. Perception, 37, 1488-1494.

Hägerhäll, C.M., T. Purcella, \& R. Taylor (2004). Fractal Dimension of Landscape Silhouette Outlines as a Predictor of Landscape Preference. Journal of Environmental Psychology. 24, 247-255.

Hraska, J. (2015). Chronobiological aspects of green buildings daylighting. Renew.Energy 73,109e114.
Hunter, M.D., Eickhoff, S.B., Pheasant, R.J., Douglas, M.J., Watts, G.R., Farrow, T.F.D., Hyland, D., Kang, J., Wilkinson, I.D., Horoshenkov, K.V., Woodruff, P.W.R. (2010). The state of tranquility: subjective perception is shaped by contextual modulation of auditory connectivity. Neuro Image 53, 611e618.

Hwang, Y.H., Yue, Z.E.J. (2015). Observation of biodiversity on minimally managed green roofs in a tropical city. J. Living Archit. 2, 9e26.

Hwang, B.G., Shan, M., Tan, E.K. (2016). Investigating reworks in green building construction projects: magnitude, influential factors, and solutions. Int. J. Environ. Res. 10 (4), 499-510. https://doi.org/10.22059/ijer.2016.59613.

Ignatius, M., Wong, N.H., Jusuf, S.K. (2015). Urban microclimate analysis with consideration of local ambient temperature, external heat gain, urban ventilation, and outdoor thermal comfort in the tropics. Sustain. Cities Soc. 19, 121e135.

Ikemi, M. (2005). The Effects of Mystery on Preference for Residential Facades. Journal of Environmental Psychology, 25, 167-173.

International well building institude (2019, October, 21). Retrieved from https://skylineartservices.com/wpcontent/uploads/Biophilic-well-buildingHandout.pdf

Jahncke, H., S. Hygge, N. Halin, A.M. Green, \& K. Dimberg (2011). Open-Plan Office Noise: Cognitive Performance and Restoration. Journal of Environmental Psychology, 31, 373-382.

Joye, Y. (2007). Architectural Lessons From Environmental Psychology: The Case of Biophilic Architecture. Review of General Psychology, 11 (4), 305-328.

Kahn, Jr. P.H., B. Friedman, B. Gill, J. Hagman, R.L. Severson, N.G. Freier, E.N. Feldman, S. Carrere, \& A. Stolyar (2008). A Plasma Display Window? The Shifting Baseline Problem in a Technology Mediated Natural World. Journal of Environmental Psychology, 28 (1), 192-199.

Kaplan, R. \& S. Kaplan (1989). The Experience of Nature: A Psychological Perspective. Cambridge: Cambridge University Press.

Kaplan, S. (1988). Perception and Landscape: 
Conceptions and Misconceptions. In J. Nasar (Ed.), Environmental Aesthetics: Theory, Research, and Applications (pp. 45- 55). Cambridge, England: Cambridge University Press.

Karmanov, D. \& Hamel, R. (2008). Assessing the restorative potential of contemporary urban environment(s). Landscape and Urban Planning 86, 115-125.

Kellert, S.R. (2008). Biophilia A2- Jorgensen sven erik . In: Fath B.D. (Ed.) Encyclopedia of Ecology. Academic Press, Oxford, pp: 462-466.

Kellert, S.R., Heerwagen, J., Mador, M. (2011). Biophilic Design: The theory, science and proctice of bringing buildings to life, John Wiley \& Sons, Hoboken, N.J.

Kellert, S.R., Heerwagen, J., Mador, M. (2008). Biophilic Design: the Theory, Science and Practice of Bringing Buildings to Life. Wiley, Hoboken, N.J., p. 385

Kim, S.Y. \& J.J. Kim (2007). Effect of fluctuating illuminance on visual sensation in a small office. Indoor and Built Environment 16 (4): 331-343.

Kim, J.T., C.J. Ren, G.A. Fielding, A. Pitti, T. Kasumi, M. Wajda, A. Lebovits, \& A. Bekker (2007). Treatment with Lavender Aromatherapy in the Post-Anesthesia Care Unit Reduces Opioid Requirements of Morbidly Obese Patients Undergoing Laparoscopic Adjustable Gastric Banding. Obesity Surgery, 17 (7), 920-925.

Kohno, M., D.G. Ghahremani, A.M. Morales, C.L. Robertson, K. Ishibashi, A.T. Morgan, M.A. Mandelkern \& E.D. London (2013) Risk-Taking Behavior: Dopamine D2/D3 Receptors, Feedback, and Frontolimbic Activity. Cerebral Cortex, bht 218. First published online: August 21, 2013

Lee, S.H. (2019). Effects of biophilic design on consumer responses in the lodging industry. International Journal of Hospitality Mnagement 83: 141-150. https://doi.org/10.1016/j.ijhm.2019.05.006

Li, Q., M. Kobayashi, H. Inagaki, Y. Wakayama, M. Katsumata, Y. Hirata, Y. Li, K. Hirata, T. Shimizu, A. Nakadai, \& T. Kawada (2012). Effect of Phytoncides from Forest Environments on Immune Function. In Q. Li (Ed.). Forest Medicine (157-167). ebook: Nova Science Publishers.
Lichtenfeld, S., Elliot, A.J., Maier, M.A., Pekrun, R. (2012). Fertile Green: Green Facilitates Creative Performance, Personality and Social Psychology Bulletin, vol. 38, pp. $784 \mathrm{e} 797$.

Malnar, J.M., Vodvarka, F. (2004). Sensory Design. University of Minnesota Press, Minneapolis.

Maes, J., Liquete, C., Teller, A., Erhard, M., Paracchini, M.L., Barredo, J.I., Grizzetti, B., Cardoso, A., Somma, F., Petersen, J.-E., Meiner, A., Gelabert, E.R., Zal, N., Kristensen, P., Bastrup-Birk, A., Biala, K., Piroddi, C., Egoh, B., Degeorges, P.,Fiorina, C., Santos-Martín, F., Naru_sevi_cius, V., Verboven, J., Pereira, H.M., Bengtsson, J., Gocheva, K., Marta-Pedroso, C., Sn€all, T., Estreguil, C., San-Miguel-Ayanz, J., P erez-Soba, M., Gr^et-Regamey, A., Lillebø, A.I., Malak, D.A., Cond_e, S., Moen, J., Czúcz, B., Drakou, E.G., Zulian, G., Lavalle, C. (2016). An indicator framework for assessing ecosystem services in support of the EU Biodiversity Strategy to 2020. Ecosyst. Serv. 17, 14e23.

Morton, J. (2015). Support occupant health with the WELL building standard. Buildings 109 (9), 1919,21 .

Oldfield, E.E., Felson, A.J., Auyeung, D.S.N., Crowther, T.W., Sonti, N.F., Harada, Y., Maynard, D.S., Sokol, N.W., Ashton, M.S., Warren, R.J., Hallett, R.A., Bradford, M.A. (2015). Growing the urban forest: tree performance in response to biotic and abiotic land management. Restor. Ecol. 23, $707 \mathrm{e} 718$.

Park, B.J., Y. Tsunetsugu, T. Kasetani, T. Morikawa, T. Kagawa, \& Y. Miyazaki (2009). Physiological Effects of Forest Recreation in a Young Conifer Forest in Hinokage Town, Japan. Silva Fennica, 43 (2), 291-301.

Parkinson, T., R. de Dear, \& C. Candido (2012). Perception of Transient Thermal Environments: Pleasure and Alliesthesia. In Proceedings of 7 th Windsor Conference, Windsor, UK.

Pawlyn, M. (2011). Biomimicry in Architecture. RIBA Publishing, London.

Petherick, N. (2000). Environmental Design and Fear: The Prospect-Refuge Model and the University College of the Cariboo Campus. Western Geography, 10 (1), 89-112.

Reeve, A., Nieberler-Walker, K., Desha, C. (2017). 


\section{Journal of \\ Design Studio \\ v:2 n:1 July 2020}

Healing gardens in children's hospitals: reflections on benefits, preferences and design from visitors' boks. Urban Fro. Urban Green, 26, 48-56.

Ruso, B., \& K. Atzwanger (2003). Measuring Immediate Behavioural Responses to the Environment. The Michigan Psychologist, 4, p. 12.

Ryan, C. (2014). An Introduction to Biophilia. (2014, November 10). Retrieved January 13, 2020, from https://blog.interface.com/what-is-biophilia/

Salimpoor, V.N., M. Benovoy, K. Larcher, A. Dagher, \& R. J. Zatorre (2011). Anatomically Distinct Dopamine Release During Anticipation and Experience of Peak Emotion to Music. Nature Neuroscience, 14 (2), 257-264.

Salingaros, N.A. (2012). Fractal Art and Architecture Reduce Physiological Stress. Journal of Biourbanism, 2 (2), 11-28.

Sanchez, J.A., Ikaga, T., Sanchez, V.A. (2018). Quantitative improvement in workplace performance through biophilic design: A pilot experiment case study. Energy \& Buildings 177: 316-328.

https://doi.org/10.1016/j.enbuild.2018.07.065

Schweitzer, M., Gilpin, L., Frampton, S. (2004). Healing spaces: elements of environmental design that make an impact on health. J. Alternative Compl. Med. 10. S-71-S-83.

Senosiain Aguilar, J. (2003). Bio-architecture. Architectural Press, Oxford (Burlington, Mass).

Shan, M., Hwang, B.-G. (2018). Green building rating systems: global reviews of practices and research efforts. Sustain. Cities Soc. 39, 172-180. https://doi.org/10.1016/j.scs. 2018.02.034

Stigsdotter, U.A. \& P. Grahn (2003). Experiencing a Garden: A Healing Garden for People Suffering from Burnout Diseases. Journal of Therapeutic Horticulture, 14, 38-48.

Stovin, V. (2009). Green Roofs and Stormwater Management. Department of Civil and Structural Engineering Pennine Water Group University of Sheffield, p. 44.

Süter, A. (2015). Art made of science Microspheres.". Retrieved January 13, 2020, from http://aysegulsuter.net/portfolio/microspheres/
Taylor, R.P., (2006). Reduction of Physiological Stress Using Fractal Art and Architecture. Leonardo, 39 (3), 245-251.

Tham, K.W. \& H.C. Willem (2005). Temperature and Ventilation Effects on Performance and Neurobehavioral- Related Symptoms of Tropically Acclimatized Call Center Operators Near Thermal Neutrality. ASHRAE Transactions, 687-698.

Tsunetsugu, Y., Miyazaki, Y., Sato, H. (2007). Physiological effects in humans induced by the visual stimulation of room interiors with different wood quantities. J. Wood Sci. 53, 11e16.

Tsunetsugu, Y. \& Y. Miyazaki (2005). Measurement of Absolute Hemoglobin Concentrations of Prefrontal Region by Near-Infrared Time-Resolved Spectroscopy: Examples of Experiments and Prospects. Journal of Physiological Anthropology and Applied Human Science, 24 (4), 469-72.

Tsunetsugu, Y., Y. Miyazaki, \& H. Sato (2007). Physiological Effects in Humans Induced by the Visual Stimulation of Room Interiors with Different Wood Quantities. Journal of Wood Science, 53 (1), 11-16.

Ulrich, R.S. (1983). Aesthetic and Affective Response to Natural Environment. In I. Altman, \& J. F. Wohlwill (Eds.), Behavior and the Natural Environment (85-125). New York: Plenum Press.

Ulrich, R.S., Simons, R.F., Losito, B.D., Fiorito, E., Miles, M.A., Zelson, M. (1991). Stress recovery during exposure to natural and urban environments. J. Environ. Psychol. 11, 201e230.

UN-HABITAT. State of the World's Cities: Harmonious Cities. London Sterling. VA Nairobi, Kenya: Earthscan. (2019, January, 8). Retriewed from

https://sustainabledevelopment.un.org/content/docu ments/11192562 alt-1.pdf

USGBC. (2013). LEED V4 Reference Guide for Building Design and Construction. U.S. Green Building Council, Washington, DC.

van den Berg, A.E., T. Hartig, \& H. Staats (2007). Preference for Nature in Urbanized Societies: Stress, Restoration, and the Pursuit of Sustainability. Journal of Social Issues, 63 (1), 79-96. 


\section{Journal of \\ Design Studio \\ v:2 n:1 July 2020}

Vessel, Edward A. (2012). New York University Center for Brain Imaging. Personal communication with the authors.

Wang, K. \& R.B. Taylor (2006). Simulated Walks through Dangerous Alleys: Impacts of Features and Progress on Fear. Journal of Environmental Psychology, 26, 269-283.

Wang, D.V. \& J.Z. Tsien (2011). Convergent Processing of Both Positive and Negative Motivational Signals by the VTA Dopamine Neuronal Populations. PLoS ONE 6(2), e17047. doi:10.1371/journal.pone.0017047.

WGBC. (2014). Health, wellbeing \& productivity in offices: the next chapter for green building. In: Council, W.G.B. (Ed.), Health, Wellbeing and Productivity. World Green Building Council, UK, p. 87.

White, M., Smith, A., Humphryes, K., Pahl, S., Snelling, D., Depledge, M. (2010). Blue space: the importance of water for preference, affect, and restorativeness ratings of natural and built scenes. $J$. Environ. Psychol. 30, 482e493.

Wigö, H. (2005). Technique and Human Perception of Intermittent Air Velocity Variation. KTH Research School, Centre for Built Environment.

Wilson E.O. (1984). Biophilia: The Human Bond With Other Soecies, Harward University Press, Cambridge.

Wilson E.O. (1993). Biophilia and the conservation ethic. In SR Kellert \& E. O. Wilson (Eds.), The Biophilia Hypothesis, 2-41.

Windhager, S., K. Atzwangera, F.L. Booksteina, \& K. Schaefera (2011). Fish in a Mall Aquarium-An Ethological Investigation of Biophilia. Landscape and Urban Planning, 99, 23-30.

Xie, X.H., Gou, Z.H. (2017). Building performance simulation as an early intervention or late verification in architectural design: same performance outcome but different design solutions. J. Green Build. 12 (1), 45-61. https://doi.org/10.3992/1552-6100. 12.1.45.

Xue F, Zhonghua G., Stephen Siu-Yu Lau, Siu Kit Lau, Kin-Hung Chung. (2019a). From biophilic design to biophilic urbanism: stakeholders' perspectives. Journal of Cleaner Production 211: 1444-1452. https://doi.org/10.1016/j.jclepro.2018.11.277

Xue, F., Stephen S.Y.L., Zhonghua G., Yifan S., Boya J. (2019b). Incorporating biophilia into green building rating tools for promoting health and wellbeing. Environmental Impact Assesment Review 76:

98-112.

https://doi.org/10.1016/j.eiar.2019.02.004

Xue, F., Gou, Z., Lau, S.S.Y., (2017). Green open space in high-dense Asian cities: site configurations, microclimates and users' perceptions. Sustain. Cities Soc. $34,114 \mathrm{e} 125$.

Ye, L., Cheng, Z., Wang, Q., Lin, H., Lin, C., Liu, B. (2015). Developments of green building standards in China. Renew. Energy 73, 115-122. https://doi.org/10.1016/j.renene. 2014.05.014.

Zadeh, R.S., Shepley, M.M.Williams, G., Chung, S.S.E. (2014). The impact of Windows and daylight on acute-care nurses' physiological, physiological and behavirol health. Herd Health Environ. Res. Des. J 7 (4), 35-61.

Zald, D.H., R.L. Cowan, P. Riccardi, R.M. Baldwin, M.S. Ansari, R. Li, E.S. Shelby, C.E. Smith, M. McHugo, \& R.M. Kessler (2008). Midbrain Dopamine Receptor Availability Is Inversely Associated with Novelty-Seeking Traits in Humans. The Journal of Neuroscience, 31 December 2008, 28(53), 14372-14378; doi: 10.1523/ JNEUROSCI.2423-08.2008

Zbaśnik-Senega_enik, M., Kuzman, M.K. (2014). Interpretations of organic architecture. Prostor 22, $290 \mathrm{e} 301$.

Zevi, B. (1959). Towards an Organic Architecture. Faber \& Faber, London.

Zou, Y., Zhao, W., Zhong, R. (2017). The spatial distribution of green buildings in China: regional imbalance, economic fundamentals, and policy incentives. Appl. Geogr. 88, 38-47. https://doi.org/10.1016/j.apgeog.2017.08.022.

Zhang, H. (2003). Human Thermal Sensation and Comfort in Transient and Non-Uniform Thermal Environments, Ph.D. Thesis, CEDR, University of California at Berkeley, http://escholarship.org/uc/item/11m0n1wt

Zhang H, E. Arens, C. Huizenga, \& T. Han (2010). Thermal Sensation and Comfort Models for Non-Uniform and Transient Environments: 
Part II: Local Comfort of Individual Body Parts.

Building and Environment, 45 (2), 389-398. 
